УДК 658.512 .2

10.17213/2075-2067-2019-4-36-43

\title{
ОПЫТ И ПЕРСПЕКТИВЫ ПРИМЕНЕНИЯ \\ СИСТЕМНОЙ ПАРАДИГМЫ Я. КОРНАИ ПРИ ПРОЕКТИРОВАНИИ \\ ПРОИЗВОДСТВЕННЫХ И ТЕХНИЧЕСКИХ СИСТЕМ
}

(C) 2019 г. Е. Б. Колбачев*, Ш. Халас**, В. Е. Федорчук*

*Южно-Российский государственный политехнический университет (НПИ), 2. Новочеркасск

**Будапештский университет технологий и экономики, Венгрия

В статье показано, что для проектирования производственных и технических систем и объектов в условиях NBIC-конвергенции требуется новая методологическая основа, гармонизирующая сочиальные, экономические и технические аспекты проектирования. В качестве такой методологической основы может служить системная парадигма, разработанная венгерским экономистом и системотехником Янотем Корнаи. Сущзность системной парадигмы состоит в том, что функционирование экономики и общества осуществление прочессов производства, распределения, обмена и потребления материальных и нематериальных благ - рассматривается через призму создания, взаимодействия и трансформачии социально-экономических систем. Предложень подходы к формированию инженерно-экономического инструментария на основе системной парадигмы.

Ключевые слова: Янош Корнаи; системная парадигма; производственные системы; технические системы; NBIC-конвергенция; человеческий капитал; сочуиальный капитал.

The article shows that the design of production and technical systems and facilities in the conditions of NBIC convergence requires a new methodological framework that harmonizes the social, economic and technical aspects of design. The system paradigm developed by the Hungarian economist and system engineer Janos Kornai can serve as such a methodological basis. The essence of a system paradigm is that economy functioning and society - processes of production, distribution, exchange and consumption of tangible and intangible benefits - is seen through the prism of creation, interaction and transformation of socio-economic systems. Approaches to the formation of engineering and economic tools based on the system paradigm are proposed.

Key words: Janos Kornai; system paradigm; production systems; technical systems; NBICconvergence; human capital; social capital.

\section{1. Введение, актуальность задачи}

Получение новых знаний об особенностях решения социально-экономических задач путем создания человекоориентированных технических и производственных (социотехнических) систем в условиях NBIC-конвергенции и формирование на их базе ме- тодологической основы, гармонизирующей социальные, экономические и технические аспекты проектирования, является непременным условием успешного развития общества в современных условиях.

Создание такой основы требует преодоления ряда методологических противоречий, 
препятствующих постановке задач проектирования на основе социальных целей, их трансформирования в экономические характеристики, на основе которых формируются организационно-технические решения.

Совершенствованию методов проектирования производственных и технических систем, особенно необходимому в условиях перехода российской промышленности к инновационной модели развития при внешних ограничениях и необходимости импортозамещения, препятствует недостаточность методических знаний о взаимодействии социальных, экономических и технических компонентов процесса создания технических систем и объектов в условиях NBIC-конвергенции. Необходимо приращение таких знаний и создание концептуального подхода к формированию инженерных решений на основе социальных целей, которое будет способствовать совершенствованию идеалов и норм научного и технического сообщества, совершенствованию инженерной этики и повышению эффективности создаваемой техники и технологии. Это будет способствовать переходу российской промышленности к новым передовым интеллектуальным и цифровым технологиям.

\section{2. Современное состояние исследований и разработок в области проектировании производственных и технических систем}

Традиционным для советского периода был подход к управлению проектированием, основанный на выполнении технического задания на проектирование, в котором социально значимые факторы и параметры практически не использовались. При этом этапы и процедуры проектирования регламентировались стандартами групп ЕСКД, ЕСТД, ЕСТПП.

В восьмидесятых годах прошлого века в СССР активизировались работы по методологии проектирования, что было связано с директивно продвигаемыми на производство методами функционально-стоимостного анализа. Однако некоторые основы управления проектированием были заложены ранее. Здесь необходимо отметить работы Ю. М. Соболева [1]. Примерно в те же годы появились работы Г.С. Альтшуллера, впоследствии переизданные за границей [2]. Кроме того, ориги- нальные подходы были характерны для работ Б. И. Кудрина [3], А.И. Половинкина [4] и др.

В начале XXI века была опубликована работа Я. Корнаи [5], на ее основе в России были выполнены исследования [6, 7, 8 и др.], в которых затрагивались отдельные вопросы методологии и организации проектирования. Это пример конструктивного и неформального международного развития научных идей, воплощенных в Венгрии и России.

Традиционными для постсоветской России являются подходы и методы управления проектированием, основанные на достижении коммерческих результатов как основных целевых критериев, в некоторых случаях сочетающихся с формализованными методами генерирования технических решений, носящих в определенной мере когнитивный характер. При этом последние опираются, как правило, на определенные методические заделы, созданные в советский период. Например, работа [9], развивающая вышеописанные идеи А.И. Половинкина; исследования [10], основанные на работах Б.И. Кудрина и др.; новые работы по проблемам функционально-стоимостного анализа и проектирования $[11,12]$. Идеи Г. С. Альтшуллера в конце XX - начале XXI века были реализованы за границей его учениками [13].

Ряд российских исследований последних десятилетий были посвящены специальным вопросам технологии и организации проектирования [14, 15].

Определенная активизация работ, посвященных методологии проектирования технических систем, наблюдается и за границей. В частности, среди работ, выполненных за пределами России по данной тематике, можно назвать исследования Ш. Халаса [16, $17,18]$.

Вопросам организации и управления проектированием человекоориентированных производственных и технических систем в условиях NBIC-конвергенции посвящено немного работ [19, 20, 21].

3. Цель, задачи и сущность новых методов управления проектированием производственных и технических систем

В свете изложенного в предыдущем разделе можно сформулировать современные цель и задачи исследований по проблемам 
проектирования производственных и технических систем, актуальные для современной России и значимые для мировой науки, техники и экономики.

Цель - разработка методологии проектирования производственных и технических систем, обеспечивающей социальные и экономические интересы общества, проявляющиеся в наращивании человеческого и социального капитала и переходе к новым передовым интеллектуальным технологиям.

Для достижения указанной цели должны быть решены следующие методологические задачи:

1) преодоление противоречий между целями, поставленными при проектировании производственных и технических систем и объектов, и достигаемыми результатами;

2) преодоление противоречий между коммерческими интересами заказчика проекта и социальными интересами общества в целом в создании производственных и технических систем и объектов;

3) преодоление противоречий между имеющимися знаниями о методах проектирования и развитием когнитивной науки, на основе достижений которой методы проектирования могут совершенствоваться;

4) преодоление противоречий между состоянием знания о технических системах на начальных этапах проектирования (включая возникновение замысла новой системы) и состоянием такого знания в периоды производства и эксплуатации системы;

5) разработка управленческого инструментария, обеспечивающего решение задач $1 . .4$ в условиях NBIC-конвергенции на основе социальных целей, их трансформирования в экономические характеристики, на базе которых формируются организационно-технические решения.

Исследования, выполненные с такими целями и задачами, будут обладать научной новизной.

Новизна научной идеи исследования заключается в создании методов проектирования технических систем на базе социальноэкономических критериев, определяемых на основе системной парадигмы и связанных с исключением (снижением вероятности) вредного воздействия создаваемой системы на человека и окружающую среду, которая обеспечивается особенностями ее конструкции.

В отличие от традиционных подходов и методов управления проектированием, основанных на достижении коммерческих результатов как основных целевых критериев, в некоторых случаях сочетающихся с формализованными методами генерирования технических решений, носящих в определенной мере когнитивный характер, предлагаемый подход носит комплексный характер и гармонизирует социальные, экономические и технические аспекты процессов проектирования производственных и технических систем.

Новизна предлагаемой постановки вопроса заключается в том, что обоснованные с использованием положений системной парадигмы стоимостные характеристики конструкции могут быть определены из экономически целесообразных затрат на обеспечение безопасной работы создаваемой технической системы, а конструирование системы осуществляется исходя из параметрической сложности элементов (подсистем) системы, рассчитанной на основе их обоснованной стоимости.

В отличие от традиционных подходов и методов управления проектированием, основанных на выполнении технического задания на проектирование, в котором социально значимые факторы и параметры используются наряду с прочими, данная постановка вопроса предполагает последовательное вовлечение в процесс проектирования социальных, экономических и технических факторов и характеристик создаваемой системы. При этом социальные факторы и характеристики являются приоритетными.

В основе предлагаемой методологии лежит системная парадигма Я. Корнаи [5], развитая в последующих исследованиях [22] — концепция видения объекта и предмета исследований, согласно которой социально-экономическое пространство рассматривается как единая система, заключающая в себе множество относительно самостоятельных подсистем, состав и структура которых определяется в соответствии с позицией наблюдателя или группы наблюдателей. Причины возникновения и распространения системной парадигмы были связаны с кризисом ортодоксальной экономической теории. При 
этом под социально-экономической системой понимается относительно обособленная и устойчивая часть или определенный аспект социально-экономического пространственно-временного континуума, для которого характерны внешняя целостность и внутреннее многообразие.

В рамках этой парадигмы любая техническая система (которая, по сути, является социотехнической, ибо предусматривает участие человека в ее создании и использовании) может рассматриваться как именно такая подсистема, находящаяся в некотором социально-экономическом пространстве.

Системная парадигма Я. Корнаи была положена в основу понимания производственной системы как мультипространственной системы, объединяющей в себе разнородные элементы и ресурсы [23]. Практически на нее опираются представления Ш. Халаса [16] об оптимизации технических систем. Результаты этих работ были использованы и развиты в достаточно многочисленных исследованиях и разработках, выполненных в основном в России [6, 7, 8 и др.] при проектировании производственных систем различного отраслевого и функционального назначения.

Некоторые из методов и подходов, рассматриваемых здесь, частично были применены при обосновании цены и конструкции локомотивов и другой железнодорожной техники, горно-шахтного оборудования, оборудования для мелиорации и водного хозяйства [24, 25] и др. Работы по развитию этих научных заделов активизировались после 2014 года, что было обусловлено актуализацией данной тематики в контексте перехода экономики России к инновационной модели развития и масштабному импортзамещению. Об их результатах было опубликованы статьи в ряде изданий $[19,20,21,26,27]$.

Таким образом, задавая в качестве основополагающих определенные социально значимые нормы и требования к создаваемым техническим системам, можно обеспечивать их ориентированность на человека - свойство, которое в условиях NBIC-конвергенции проявляется специфически.

NBIC-конвергенция, являющаяся основной тенденцией современного технологического развития, предоставляет значительные возможности для роста производительности и эффективности производства и экономического роста.

В условиях NBIC-конвергенции человеческий капитал при создании технологий и производственных систем наращивается значительно в большей степени, чем в традиционных технологиях и производственных системах.

Причины этого:

1) NBIC-технологии основываются на результатах фундаментальных и поисковых исследований. Эти исследования выполняются высококвалифицированными исследователями и являются основным источником новых знаний;

2) высокая сложность биологических, наноразмерных и информационных процессов требует высокой квалификации специалистов, проводящих прикладные исследования и разрабатывающих технологии;

3) особое внимание, уделяемое когнитивным процессам, непосредственно связанным с познавательной деятельностью человека и генерированием новых технологических и других решений;

4) сложность NBIC-технологий требует создания специального оборудования, из которого комплектуются уникальные производственные системы. Проектирование, изготовление и эксплуатация этого оборудования требуют работников высокой квалификации;

5) особое внимание безопасности NBICтехнологий (в части воздействия на природную среду и человека) требует выполнения специальных исследований. Они проводятся специалистами высокой квалификации и являются дополнительными источниками новых знаний.

Социальный капитал общества благодаря NBIC-конвергенции также получает дополнительные возможности для роста.

Причины этого:

1) повышенное внимание к надежности и антропологической безопасности NBIC-технологий делает практически неизбежным общественное обсуждение с привлечением специалистов, общественных деятелей, политиков, СМИ. Это будет способствовать повышению степени доверия в обществе и, соответственно, наращиванию социального капитала;

2) в исследованиях и разработках по проблемам NBIC-конвергенции участвуют пред- 
ставители различных областей знаний. Это делает необходимым их тесное взаимодействие, взаимное доверие и существенно снижает вероятность оппортунистического поведения, что также служит наращиванию социального капитала;

3) результаты сопутствующих NBIC-конвергенции исследований в области аналитической философии и психологии по проблеме «сознание и мозг» mind-brain problem способствуют развитию прикладных поведенческих наук behavioral science. Эти науки служат в числе прочего гармонизации взаимоотношений отдельного человека и общества и тем самым способствуют наращиванию социального капитала.

Признавая перспективы развития NBICтехнологий, многие исследователи $[20,28]$ указывают на риски и угрозы, обусловленные их широким распространением Эти риски и угрозы связываются, главным образом, с опасностями воздействия на человека, его биологического и психологического изменения.

Говоря об ориентированности на человека и безопасности технической системы (изделия), необходимо иметь в виду два аспекта: безаварийность работы (так как любая авария с большей или меньшей вероятностью наносит вред здоровью и угрозу жизни человека) и экологическое благополучие, предполагающее отсутствие вреда здоровья человека при устойчивой работе создаваемой технической системы.

При оценке результатов деятельности проектируемых объектов или технических систем по степени снижения рисков сущность процессов, связанных с их функционированием, может быть описана с помощью моделей страхования, а соответствующие стоимостные параметры могут быть определены с помощью актуарных расчетов. При этом в качестве аналога (альтернативы) деятельности проектируемого объекта/системы рассматривается вариант массового рискового страхования - страхования, охватывающего значительное число субъектов и рисков, характеризующихся однородностью объектов и незначительным разбросом в размерах страховых сумм.

Таким образом, в качестве рабочей модели рассматривается ситуация, при которой экономический результат функционирования проектируемого объекта/системы полагается идентичным результату создания специального страхового фонда, позволяющего проводить мероприятия по преодолению чрезвычайных ситуаций, обусловленных прекращением существования этого объекта/системы.

Аналогом страхового тарифа (тарифа страховых взносов) выступает экономически обоснованный размер затрат на функционирование проектируемого объекта/системы. При этом предполагается, что ежегодно на его содержание необходима сумма, равная страховым платежам, которые должны вноситься, чтобы компенсировать ущерб, обусловленный прекращением существования проектируемого объекта.

При обосновании расходов на содержание проектируемого объекта рассчитываются следующие показатели: частота событий, коэффициент кумуляции риска и другие страховые параметры, определяемые на основе актуарной модели.

В рассматриваемом случае расчет может выполняться исходя из того, что величина суммы собранных страховых взносов идентична значению эксплуатационных расходов на содержание проектируемого объекта за расчетный период, а сумма выплаченного страхового возмещения - значению затрат на преодоление кризисных ситуаций. При этом в качестве общей страховой суммы застрахованных объектов должна быть принята величина, равная совокупным затратам на преодоление кризисной ситуации и определенная из предположения, что будет компенсирована также определенная часть недополученного дохода. При прогнозировании уровня опасности проектируемых объектов, относящихся к группам изделий, по которым нет статистической информации об аварийных ситуациях, возникающих в ходе их эксплуатации, могут быть применены положения теория решений, которая дополняется формулой Байеса.

На основе целесообразных затрат на функцию обеспечения безопасной работы с использованием метода стоимостного проектирования может быть рассчитана сумма расходов на эксплуатацию создаваемой технической системы/объекта и обоснована ее цена. Эти параметры после согласования с заказчиком становятся основополагающи- 
ми при разработке конструкции технической системы/объекта.

Для практической реализации вышеописанной концепции должны быть разработаны методы и иинструментарий для социально-экономического управления созданием технических систем на основе их функций и параметров при техническом проектировании и рабочем проектировании. При этом установленная и согласованная с заказчиком стоимость создаваемой машины или иной технической системы служит ориентиром при формировании конструкции на этапах технического и рабочего проектирования, в ходе которого параметры технической системы определяются на основе ее функциональной модели.

Социальным результатом проектирования технической системы служит прирост человеческого и социального капитала в результате проектирования, изготовления и эксплуатации технической системы, которые могут быть оценены на основании параметрической сложности и профессионального тезауруса работников, занятых проектированием, изготовлением и эксплуатацией технической системы

\section{4. Заключение}

Для проектирования производственных и технических систем и объектов в условиях NBIC-конвергенции требуется новая методологическая основа, гармонизирующая социальные, экономические и технические аспекты проектирования.

В качестве такой методологической основы может служить системная парадигма, разработанная венгерским экономистом и системотехником Яношем Корнаи. Сущность системной парадигмы состоит в том, что функционирование экономики и общества - осуществление процессов производства, распределения, обмена и потребления материальных и нематериальных благ - рассматривается через призму создания, взаимодействия и трансформации социально-экономических систем.

Это позволит получить приращение знаний о взаимодействии социальных, экономических и технических компонентов процесса создания технических систем и объектов в условиях NBIC-конвергенции. Концепту- альный подход к формированию инженерных решений на основе социальных целей будет способствовать совершенствованию идеалов и норм научного и технического сообщества, совершенствованию инженерной этики.

Обоснованные с использованием положений системной парадигмы стоимостные характеристики конструкции могут быть определены из экономически целесообразных затрат на обеспечение безопасной работы создаваемой технической системы, а конструирование системы осуществляется исходя из параметрической сложности элементов (подсистем) системы, рассчитанной на основе их обоснованной стоимости.

\section{Литература}

1. Соболев Ю.М. Конструктор выбирает решение. - Пермь: Книжн. изд-во, 1964.

2. Altshuller G. Creativity as an Exact Science. - London: Gordon and Breach, Science Publishers Inc., 1984.

3. Кудрин Б.И. Введение в технетику. Томск: Изд-во ТГУ, 1991. - 524 с.

4. Половинкин А.И. Основы инженерного творчества. - М.: Машиностроение, 1988. - 294 c.

5. Корнаи Я. Системная парадигма // Вопросы экономики. - 2002. - №4. - С. 18-26.

6. Колбачев Е.Б. Глобализация, информациональная экономика и российские предприятия // Изв. вузов Сев.-Кавк. регион. Общественные науки. - 2002. - Прил. №1. C. 29-39.

7. Передерий М.В., Ткалич Г.И. Системотехнические основы проектирования организационных структур бизнес-групп и других консолидационных группировок на основе единой инфраструктуры // Вестник Южно-Российского государственного технического университета (Новочеркасского политехнического института). Серия: Сoциально-экономические науки. - 2018. №1. - С. 20-25.

8. Боровая Л.В. Системная парадигма как основа управления развитием политехнического университета // Вестник ЮжноРоссийского государственного технического университета (Новочеркасского политехнического института). Серия: Социально-экономические науки. - 2018. - №4. - С. 16-20. 
9. Zaboleeva-Zotova A. V., Bobkov A.S., Orlova Y.A., Rozaliev V.L. Automated identification of human emotions based on analysis of body movements / Proceedings of the IADIS International Conferences - Interfaces and $\mathrm{Hu}$ man Computer Interaction 2013, IHCI 2013 and Game and Entertainment Technologies 2013. P. 299-304.

10. Кузьминов А.Н. Ценозы в контексте эволюционного подхода // Вестник Российского экономического университета им. Г. В. Плеханова. - 2019. - №1 (103). C. $120-134$.

11. Ковалев А.П., Рыжжова В.В. Основы стоимостного анализа. - М.: Финансы и статистика, 2007.

12. Применение функционально-стоимостного анализа в решении управленческих задач / Под ред. В.В. Рыжовой. - М.: ИНФPA-M, 2011. - $245 \mathrm{c}$.

13. Zlotin B., Zusman A., Hallfell F. TRIZ to invent your future utilizing directed evolution methodology // Procedia Engineering. 2011. - Vol. 9. - P. 126-134.

14. Ярушкина Н.Г. Автоматизированное проектирование сложных технических систем в условиях неопределенности /дисс. докт. техн. наук. - 2012.

15. Султанова И.П. Проекты организации производства на основе экономико-визуальной модели / дисс. докт. техн. наук. 2009.

16. Halasz S., StumpfP. Optimization of PWM for the Overmodulation Region of TwoLevel Inverters // IEEE transactions on industry application. - 2018. - Vol. 54, Issue 4. P.3393-3404.

17. Halasz S., Stumpf P. Optimal PWM for Two-level Inverter fed High Speed Induction Machines / 2018 IEEE $18^{\text {th }}$ International Power Electronics and motion control conference (PEMC). - P. 1012-1018.

18. Stumpf P., Halasz S. Comparison of Optimized PWM Techniques for Overmodulation Region of Two-level Inverters / $17^{\text {th }}$ International Ural conference on $\mathrm{AC}$ electric drives (ACED). - 2018.
19. Kolbachev E., Kolbacheva T., Salnikova $Y$. Production in the condition of NBIC-convergence: the role of socio-emotional and cognitive skills / EDUHEM 2018 VIII International conference on intercultural education and International conference on transcultural health.

20. Kolbachev E., Kolbacheva T. Biological and social factors that exert an impact on decision making during working-out of the convergent technologies // Advances in Intelligent Systems and Computing. - 2018. - Vol. 722. - P. 255-260.

21. Kolbachev E., Kolbacheva T. Human Factor and Working Out of NBIC Technologies // Advances in Intelligent Systems and Computing. - 2018. - Vol. 793. - P. 179-190.

22. Клейнер Г. Системная парадигма и теория предприятия // Вопросы экономики. 2002. — №10. - C. 24-33.

23. Колбачев Е.Б. Производственные системы машиностроительных предприятий и их организационно-экономическая эволюция // Проблемы машиностроения и автоматизации. - 2003. - №2. - С. 12-16.

24. Gorobets D. G. Economic features of working out dangerous or responsible products of machine-building / B cб.: Cost analysis and innovation of the enterprise. - Novocherkassk: SRSTU (NPI), 2000. - P. 23-24.

25. Liderman K.M. Economic instruments to work out a really efficient mining equipment / В сб.: Economy of the business processes and production system. - Novocherkassk: SRSTU (NPI), 2002. - P. 25-28.

26. Kolbachev E., Perederiy M., Salnikova $Y$. The Cost of Ensuring the Safety of Technical Systems and Their Service Life // Advances in Intelligent Systems and Computing II. 2019. - Vol. 1026. - P. 573-578.

27. Kolbachev E., Salnikova Y. Actuarial Models in the Design of Human-oriented Production Systems and Products // Advances in Intelligent Systems and Computing. — 2019. Vol. 1018. - P. 163-169.

28. Dubrovski D.I. Subjective reality and the brain: an essay on a theoretical solution to the problem // Her. Russ. Acad. Sci. - 2013. Vol. 1. - P. 59-69. 


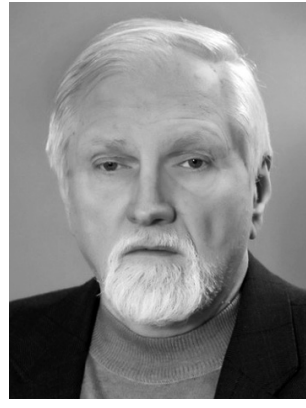

Колбачев Евгений Борисович - доктор экономических наук, кандидат технических наук, профессор, заведующий кафедрой «Производственный и инновационный менеджмент» Южно-Российского государственного политехнического университета (НПИ).

Kolbachev Evgueniy Borisovich - Doctor of Economics, Candidate of Engineering, Professor, head of «Production Management and Management of the Innovations» department of the SouthRussian state Polytechnic University (NPI).

346428 , г. Новочеркасск, ул. Просвещения, 132

132 Prosveshcheniya st., 346428, Novocherkassk, Russia

Тел.: +7 (8635) 25-56-66, +7 (8635) 25-51-54

E-mail: kolbachev@yandex.ru

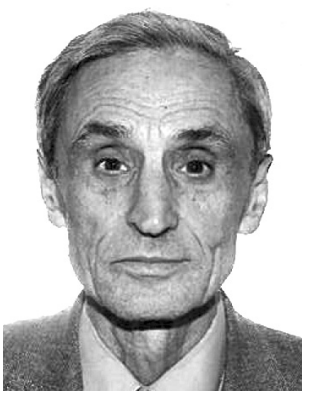

Халас Шандор - доктор технических наук, профессор кафедры электроэнергетики Будапештского университета технологий и экономики.

Halas Shandor - doctor of technical Sciences, Professor of the Department of electric power engineering of Budapest University of technology and Economics.

1111, г. Будапешт, Венгрия, Müegyetem rakpart 1-6

Müegyetem rakpart 1-6, 1111, Budapest, Hungary

Тел.: +36 1 463-11-11, факс: +36 1 463-11-10; e-mail: info@bme.hu

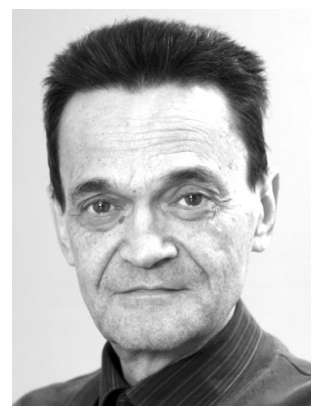

Федорчук Владимир Евгеньевич - доцент кафедры «Производственный и инновационный менеджмент» ЮжноРоссийского государственного политехнического университета (НПИ).

Fedorchuk Vladimir Evguenievich - associate Professor of «Production Management and Management of the Innovations» department of the South-Russian state Polytechnic University (NPI).

346428, г. Новочеркасск, ул. Просвещения, 132

132 Prosveshcheniya st., 346428, Novocherkassk, Russia

Тел.: +7 (8635) 25-56-66, +7 (8635) 25-51-54; e-mail: fiop_urgtu@mail.ru 